\title{
Fast Mesoscopic Simulation Of Grain Growth And Macroscopic Modeling.
}

\author{
Daniel Weisz-Patrault ${ }^{1, a)}$, Sofia Sakout ${ }^{1}$ and Alain Ehrlacher ${ }^{2}$ \\ ${ }^{1}$ LMS, CNRS, École Polytechnique, Institut Polytechnique de Paris, F-91128 Palaiseau, France. \\ ${ }^{2}$ Navier, CNRS, École des Ponts ParisTech, 6 \& 8 Ave Blaise Pascal, 77455 Marne La Vallee, France. \\ a) Corresponding author: daniel.weisz-patrault@ polytechnique.edu \\ ${ }^{b}$ URL: www.polytechnique.edu/annuaire/en/users/daniel.weisz-patrault
}

\begin{abstract}
The Orientated Tessellation Updating Method (OTUM) is a fast mesoscopic model of grain growth that has been published recently for a single phased metal without diffusion or segregation of alloying elements. It is based on tessellation techniques and simple linear algebra. On this basis, some ideas to develop an upscaling strategy are broached in this work. The aim is to develop a macroscopic model of grain growth whose state variables contain for each material point the statistical descriptors of the microstructure (e.g., disorientation, grain size and shape distributions). The approach consists in reducing the amount of data needed in the mesoscopic model. Indeed, state variables of the mesoscopic model lies in a high-dimensional space (several hundreds of dimensions) although state variables of the corresponding macroscopic model should ideally be in a low-dimensional space (e.g., less than 10 dimensions). The strategy is to determine macroscopic free energy and dissipation potentials on the basis of a large number of computations at the mesoscopic scale (i.e., polycrystal). In addition, the mesoscopic model is perfectly deterministic. However, as a macroscopic state may corresponds to a large number of different mesoscopic states an epistemic randomness arises at the macroscopic scale.
\end{abstract}

\section{MESOSCOPIC MODEL}

Recently, a fast mesoscopic model of grain growth has been developed [1] (see Figure 1) and is called the Orientated Tessellation Updating Method (OTUM). Although the method is general, the model has been presented for plane polycrystals and single phased metal without diffusion or segregation of alloying elements. This model is sufficiently simple and fast to enable a large number of simulations of various microstructures, even if it leads to neglect some phenomena occurring at this scale. Usual grain growth models relying on cellular automaton [2-5], mobile finite element modeling [6], level set functions [7, 8], phase field [9-12] or molecular dynamics [13-15] have been intensively studied and very interesting results have been obtained. However, the computational cost of such approaches is usually incompatible with an intensive use as suggested within the proposed framework. Vertex methods [16-19] consist in establishing the evolution law directly at the triple junctions and are sufficiently simple in two dimensions to reach short computation time. However, the extension in three dimensions is difficult.

Evolution of polycrystalline microstructures (such as grain growth) involves coupled mechanisms at different scales: (i) the atomic scale (crystal lattice), (ii) the microscale including mechanisms at grain boundaries (iii) the mesoscale revealing the grain structure (i.e., polycrystalline Representative Volume Element) and (iv) the macroscopic scale modeled as a continuous medium. Thus, the OTUM has been developed as a "toy" model formulated at mesoscale and enabling to simulate grain growth within short computation time.

To achieve such computational efficiency, fast tessellation techniques underlie the method. Indeed, VoronoiLaguerre tessellation techniques are usually used to approximate polycrystalline microstructures at mesoscale. Very efficient algorithms have been developed with the possibility of controlling statistical distributions of grain size and shape. Crystal lattice orientation can also be specified for each grain. The tessellation equipped with such an orientation field is called an Orientated Tessellation (OT). Thus, the real evolution of the microstructure can be approximated as a succession of OT approximations. The modeling strategy proposed in [1] (OTUM) consists in deriving the evolution law of the microstructure directly on the parameters defining the OT. Thus, the state variables of this mesoscopic 
model consist of all the parameters needed to define an OT (i.e., seeds positions, weights, crystal orientations). Thus, for a plane polycrystal of only 500 grains, there are around 2000 state variables. Even though the number of state variables is fairly reduced in comparison with other numerical approaches (level set functions etc.), one cannot process such a high number of degrees of freedom for each material point of a macroscopic model. Thus, to study the grain growth of macroscopic structures (e.g., fabrication and forming processes) it is necessary to reduce the amount of data.

As grain growth during annealing (grain coarsening) is essentially viscous, the time derivatives of the OT parameters are obtained as a function of the thermodynamic forces defined as the partial derivatives of the total grain boundary energy with respect to the OT parameters. This mesoscale evolution law is obtained through the energy balance equation by specifying two mechanisms at microscale: (i) the grain boundary energy (disorientation) and (ii) the dissipated power by crystal visco-plasticity through any grain boundary virtual motion. In addition, plane polycrystals are considered with three slip directions in each grain. Thus, the crystal lattice is plane hexagonal. This configuration corresponds to the plane $\langle 1,1,1\rangle$ of a face-centered cubic (fcc) crystal. Disorientation between two neighboring grains (characterized by five parameters in 3D) is characterized only by two parameters in 2D: the disorientation angle $\Delta \theta$ and the orientation of the grain boundary $\varphi$. Thus, the grain boundary energy is computed from fcc crystals sharing the same orientation $\langle 1,1,1\rangle$ (asymmetric tilt boundaries). The dissipative mechanism at microscale is calibrated to correspond to the classic notion of grain boundary mobility.

Mathematical derivations to obtain the mesoscopic evolution law rely on simple linear algebra. More precisely, each cell (denoted by $C_{j}$ where $1 \leq j \leq n$ with $n$ the number of cells) of the Voronoi-Laguerre tessellation is defined as follows:

$$
C_{j}=\left\{\left(\begin{array}{l}
x \\
y
\end{array}\right) \in \mathbb{R}^{2}, \forall k \in\{1, \cdots, n\},\left\|\begin{array}{c}
x-x_{j} \\
y-y_{j}
\end{array}\right\|^{2}-w_{j} \leq\left\|\begin{array}{c}
x-x_{k} \\
y-y_{k}
\end{array}\right\|^{2}-w_{k}\right\}
$$

where $\left(x_{j}, y_{j}\right) \in[0,1]^{2}$ are the dimensionless Cartesian coordinates of the seeds and $w_{j}$ are the weights. Since the weights are defined up to a constant an additional constraint is added so that the definition is univocal:

$$
\sum_{j=1}^{n} w_{j}=1
$$

So the vector of weights $\boldsymbol{w}=\left(w_{1}, \cdots, w_{n}\right)$ lie in an affine hyperplane of dimension $n-1$ and denoted by $P_{a}^{(n-1)}=$ $\left\{\boldsymbol{w} \in \mathbb{R}^{n}, \sum_{j=1}^{n} w_{j}=1\right\}$, whose support is the hyperplane denoted by $P^{(n-1)}=\left\{\boldsymbol{w} \in \mathbb{R}^{n}, \sum_{j=1}^{n} w_{j}=0\right\}$. Therefore the parameter set defining the OT reads:

$$
\mathcal{P}_{\mathrm{OT}}=\left\{\boldsymbol{\alpha}=(\boldsymbol{x}, \boldsymbol{y}, \boldsymbol{w}, \boldsymbol{\theta}) \in \mathbb{R}^{n} \times \mathbb{R}^{n} \times P_{a}^{(n-1)} \times\left[0, \frac{\pi}{3}\right]^{n}\right\}
$$

where $\boldsymbol{\theta}=\left(\theta_{1}, \cdots, \theta_{n}\right)$ are the orientations of the grains and $\boldsymbol{x}=\left(x_{1}, \cdots, x_{n}\right)$ and $\boldsymbol{y}=\left(y_{1}, \cdots, y_{n}\right)$ are the coordinates of the seeds. From the global energy balance and the principle of maximum dissipation the evolution law of the weights reads:

$$
\boldsymbol{R}(\boldsymbol{\alpha}) \cdot \dot{\boldsymbol{w}}=-\frac{m(T)}{L_{0}^{3}} \frac{\partial \mathcal{E}(T, \alpha)}{\partial \boldsymbol{w}}
$$

where $\boldsymbol{R}$ is a dimensionless second order tensor of size $n \times n, m(T)$ is a grain mobility, $E$ is the energy of grain boundaries and $L_{0}$ is the physical length of the tessellation. Because of the constraint (2), $\dot{\boldsymbol{w}}$ lie in $P^{(n-1)}$ (of dimension $n-1)$ and therefore the second order tensor $\boldsymbol{R}$ is not invertible (rank $n-1)$ and its $\operatorname{kernel}$ is $\operatorname{ker}(\boldsymbol{R})=(1, \cdots, 1) \mathbb{R}$. That is to say that $\operatorname{ker}(\boldsymbol{R}) \perp P^{(n-1)}$. The evolution law can therefore be inverted by using $\boldsymbol{R}^{\dagger}$ the Moore-Penrose pseudo-inverse of $\boldsymbol{R}$, and the evolution law reads:

$$
\dot{\boldsymbol{w}}=-\frac{m(T)}{L_{0}^{3}} \boldsymbol{R}^{\dagger}(\boldsymbol{\alpha}) . \frac{\partial \mathcal{E}(T, \boldsymbol{\alpha})}{\partial \boldsymbol{w}}
$$

Technically this evolution is integrated through an explicit scheme with an adaptive step-size. 

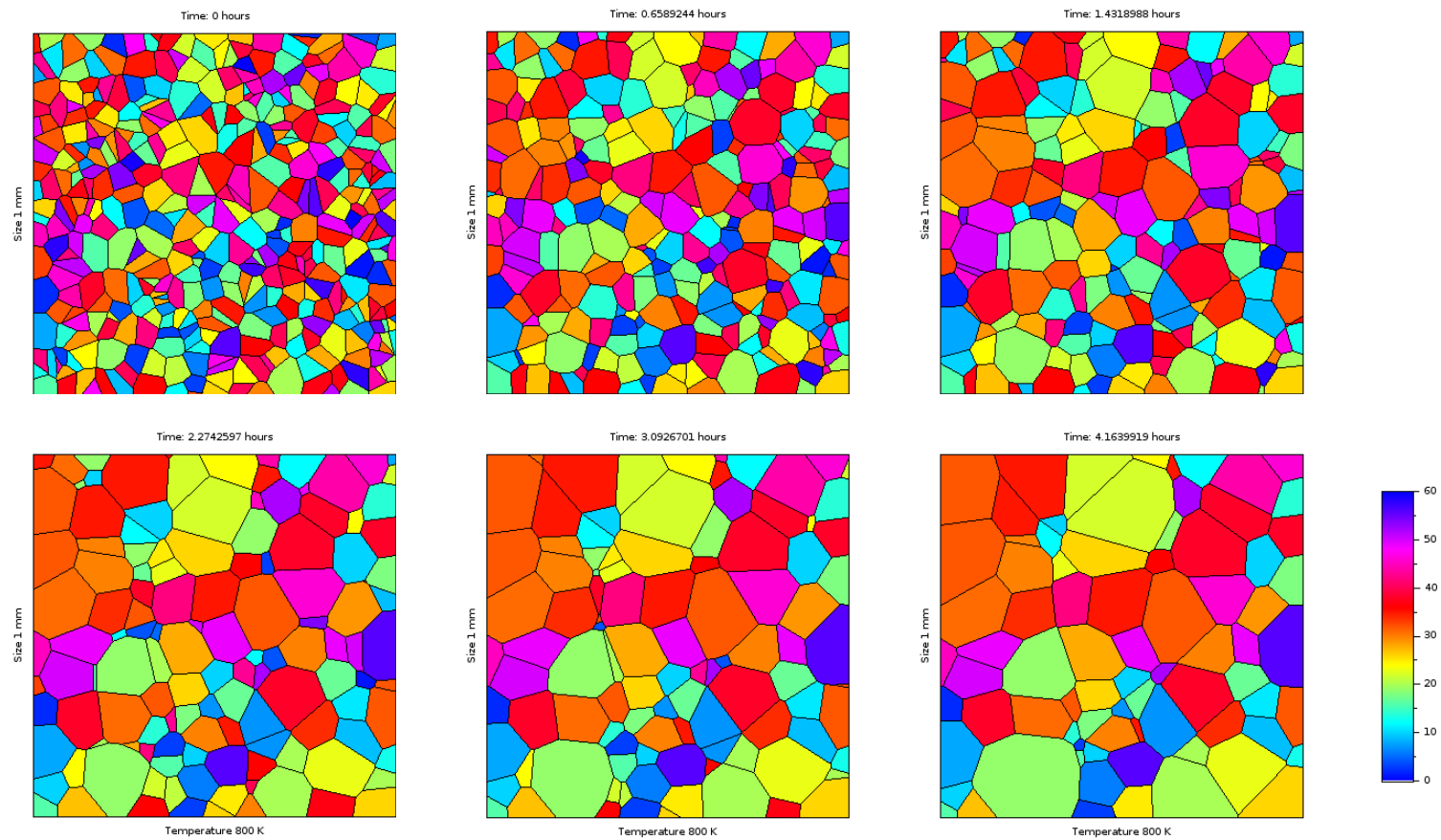

FIGURE 1. Grain growth extracted from [1].

\section{MACROSCOPIC MODEL}

This section deals with a macroscopic model of grain growth whose state variables contain for each material point the statistical descriptors of the microstructure (e.g., disorientation, grain size and shape distributions). Statistical distributions are too much detailed to be processed at each material point, thus reduced information is considered instead: means and variances at each material point. This ambition arises as very few information about microstructure is usually processed at the macroscopic scale. The general framework is the standard generalized media characterized by a free energy per unit mass and a dissipated power potential. These two potentials arise in the balance energy equation combining the first and second laws of thermodynamics. The balance energy equation should be verified for all possible virtual evolution. The two potentials depend on macroscopic state variables and their time derivatives. The determination of the macroscopic free energy and dissipation potentials enables to establish the evolution laws of the state variables characterizing the microstructure evolution at macroscale.

The strategy is to determine macroscopic free energy and dissipation potentials not axiomatically (with parametric functions) and calibration with experiments at macroscale, but on the basis of a large number of computations at the scale of the polycrystal (mesoscopic model). The aim is to quantify for each microstructure evolution the total grain boundary energy and the total dissipated power as a function of the macroscopic state variables that characterize the statistical distribution of the microstructure in order to determine the macroscopic evolution law.

In addition, the mesoscopic model is perfectly deterministic. However, as a macroscopic state may corresponds to a large number of different mesoscopic states, an epistemic randomness arises at the macroscopic scale. The macroscopic energy potential is not significantly affected by this variability and can be reasonably modeled as a deterministic potential. However, the macroscopic dissipation potential is significantly affected, that is to say that several identical virtual evolutions of the macroscopic state variables lead to very different macroscopic virtual dissipated powers. Thus, the dissipation potential should be modeled as a random variable. Then, the identification involves a Bayesian regression and the dissipation potential is given as a probability density function, which is conditional on the macroscopic state variables (explanatory variables). From a mathematical point of view this statistical upscaling method relies on (i) a deterministic identification of the energy potential from data points obtained with the mesoscopic model, (ii) a 
minimization under linear constrains to identify the data points of dissipated power through any virtual evolution of macroscopic state variables and (iii) a probabilistic identification of the dissipative potential.

\section{REFERENCES}

[1] D. Weisz-Patrault, S. Sakout, and A. Ehrlacher, 24 ${ }^{\text {ème }}$ Congrès Franais de Mécanique (2019).

[2] D. Kandel and E. Domany, Journal of Statistical Physics 58, 685-706 (1990).

[3] E. A. Holm, G. N. Hassold, and M. A. Miodownik, Acta Materialia 49, 2981-2991 (2001).

[4] E. A. Holm, M. A. Miodownik, and A. D. Rollett, Acta Materialia 51, 2701-2716 (2003).

[5] L. Zhang, A. D. Rollett, T. Bartel, D. Wu, and M. T. Lusk, Acta Materialia 60, 1201-1210 (2012).

[6] J. Gruber, D. C. George, A. P. Kuprat, G. S. Rohrer, and A. D. Rollett, Scripta materialia 53, 351-355 (2005).

[7] H. Hallberg, Modelling and Simulation in Materials Science and Engineering 22, p. 085005 (2014).

[8] B. Scholtes, R. Boulais-Sinou, A. Settefrati, D. P. Muñoz, I. Poitrault, A. Montouchet, N. Bozzolo, and M. Bernacki, Computational Materials Science 122, 57-71 (2016).

[9] N. Ma, A. Kazaryan, S. Dregia, and Y. Wang, Acta Materialia 52, 3869-3879 (2004).

[10] C. Krill Iii and L.-Q. Chen, Acta materialia 50, 3059-3075 (2002).

[11] L. Vanherpe, N. Moelans, B. Blanpain, and S. Vandewalle, Computational Materials Science 50, 2221-2231 (2011).

[12] K. Chang and N. Moelans, Acta Materialia 64, 443-454 (2014).

[13] M. Upmanyu, D. Srolovitz, L. Shvindlerman, and G. Gottstein, Acta materialia 47, 3901-3914 (1999).

[14] M. Upmanyu, D. Srolovitz, L. Shvindlerman, and G. Gottstein, Acta materialia 50, 1405-1420 (2002).

[15] M. Upmanyu, D. J. Srolovitz, A. Lobkovsky, J. A. Warren, and W. Carter, Acta Materialia 54, 1707-1719 (2006).

[16] F. Humphreys, Materials Science and Technology 8, 135-144 (1992).

[17] F. Wakai, N. Enomoto, and H. Ogawa, Acta Materialia 48, 1297-1311 (2000).

[18] M. Syha and D. Weygand, Modelling and Simulation in Materials Science and Engineering 18, p. 015010 (2009).

[19] A. Vondrous, M. Reichardt, and B. Nestler, Modelling and Simulation in Materials Science and Engineering 22, p. 025014 (2014). 